\title{
Fatores que influenciam a qualidade de vida do cuidador familiar do idoso com demência
}

\author{
Factors influencing the quality of life of family caregivers \\ of the elderly with dementia
}

Lírica Salluz Mattos Pereira ${ }^{1}$

Sônia Maria Soares ${ }^{1}$

${ }^{1}$ Escola de Enfermagem,

\begin{abstract}
The progression of symptoms of dementia leads to loss of autonomy and independence of the elderly. This progression suggests that a family member will assume the functions of caregiver, which can adversely affect his/her quality of life $(\mathrm{QOL})$. This research is an integrative review that sought to analyze the available evidence on the factors that influence quality of life of family caregivers of the elderly with dementia. Articles published in the BDENF, Lilacs and Medline databases were examined and 477 studies were selected, of which 11 met the inclusion criteria and comprised the sample of this review. It was found that the factors that influence quality of life of caregivers are: depression; poor sleep quality; dementia type and neuropsychiatric symptoms; support, social support and access to health services; leisure; pre-existing health problems; interventions for caregiver training and spirituality. It is suggested that the nursing professional identifies the needs of the caregivers, such that strategies of care can be defined.
\end{abstract}

Key words Quality of life, Caregivers, Dementia, Nursing
Resumo A piora do quadro clínico na demência leva a perda da autonomia e independência do idoso. Tal contexto pressupõe que algum familiar irá assumir as funções de cuidador, o que pode interferir na sua qualidade de vida $(\mathrm{QV})$. A presente investigação é uma revisão integrativa que teve como objetivo analisar as evidências disponiveis sobre os fatores que influenciam a $\mathrm{QV}$ do cuidador familiar do idoso com demência. Foram selecionados artigos publicados nas bases dados BDENF, Lilacs e Medline, tendo sido identificados 477 estudos, dos quais 11 atenderam aos critérios de inclusão e compuseram a amostra desta revisão. Identificou-se que os fatores que influenciam a QV desse cuidador são: depressão; má qualidade do sono; tipo de demência e sintomas neuropsiquiátricos; apoio, suporte social e acesso aos serviços de saúde; lazer; problemas de saúde pré-existentes; intervenções com treinamento para o cuidador e espiritualidade. Sugere-se que o profissional enfermeiro identifique necessidades desse cuidador, para que assim possa traçar estratégias de cuidado. Palavras-chave Qualidade de vida, Cuidadores, Demência, Enfermagem 


\section{Introdução}

O envelhecimento nas últimas décadas é um processo de magnitude mundial, destacam-se nesse processo os países de baixa e média renda. Estima-se que, em 2025, teremos aproximadamente 840 milhões de idosos, representando $70 \%$ do total de pessoas no mundo ${ }^{1,2}$.

Em adição a essa realidade emergente, $o$ envelhecimento populacional traz implicações importantes para a sociedade, tanto nas políticas públicas e sistemas de saúde, quanto no mercado de trabalho e dinâmica familiar.

Neste contexto, a demência vem assumindo papel de destaque entre as doenças crônicas e incapacitantes mais frequentes nos idosos, sendo responsável por mais de $12 \%$ dos anos vividos com incapacidades. Projeções apontam para o ano de 2025, um quantitativo de aproximadamente 34 milhões de pessoas no mundo com diagnóstico de demência ${ }^{3}$,com destaque para a doença de Alzheimer (DA) que representa 50 a $60 \%$ do número total dos casos ${ }^{4,5}$. Em sequência há a demência vascular (DV), segunda mais prevalente; a demência de Corpos de Lewy (DCL), que acomete cerca de $20 \%$ dos pacientes; a demência fronto-temporal (FTD), corresponde de $10 \%$ a 15\% dos casos; e a demência mista, que é caracterizada pela ocorrência simultânea de eventos característicos de DA e $\mathrm{DV}^{6}$.

A demência é uma doença crônica e progressiva que envolve declínio cognitivo e alterações comportamentais, comprometendo as atividades de vida diária $\left(A V D\right.$ 's) ${ }^{7}$. A piora do quadro clínico pode acarretar em perda da autonomia e total dependência, evoluindo com complicações clínicas em função da imobilidade progressiva, disfagia e desnutrição ${ }^{8}$. Dessa forma, a demência atualmente é uma das principais doenças que causam incapacidade nos idosos?.

A perda de independência do idoso pressupõe que algum membro do núcleo familiar irá assumir as funções de cuidador. Dessa forma, o cuidador familiar, ou seja, aquele membro familiar responsável por cuidar de uma pessoa da sua própria família assume essa responsabilidade, porém este cuidar pode se tornar uma atividade desgastante que propicia fatores de risco à saúde desse cuidador ${ }^{10}$.

Geralmente o cuidado é delegado a uma pessoa que possui outras tarefas e necessita conciliá-las. Este acúmulo de tarefas pode impactar na saúde do cuidador, pois ele experimenta uma sobrecarga de atividades cotidianas que implica em maior risco de morbidade psiquiátrica e física ${ }^{11,12}$. Nesse contexto ocorre um redimensionamento na vida desse cuidador para que dessa forma ele conviva com as implicações causadas pela doença do seu familiar, fatos que conduzem à significativa interferência na sua $\mathrm{QV}^{11,13}$.

Os cuidadores familiares mencionam que a experiência de assumir o cuidado a um idoso dependente é uma tarefa exaustiva, tanto no que tange aos aspectos emocionais, devido ao envolvimento afetivo, quanto às próprias restrições em vários campos de sua vida, propiciada pela readequação de sua rotina modificada para suprir as necessidades do seu familiar idoso ${ }^{14}$.

Entretanto, dentro do escopo literário, é premente direcionarmos atenção aos cuidadores familiares, muitas vezes negligenciada em nossas ações assistenciais. Observa-se que diante do ônus relacionado ao cuidado do idoso dependente, o cuidador necessita de atenção, enfoque criterioso dos serviços de saúde e capacitação, para que dessa forma consiga prestar o cuidado de maneira adequada e com o menor risco para a sua saúde física e psíquica ${ }^{14}$. Para tanto, delineou-se a presente investigação com o objetivo de analisar as evidências disponíveis sobre os fatores que influenciam a QV do cuidador familiar do idoso com demência.

\section{Método}

Trata-se de uma revisão integrativa de literatura, considerada como um método de pesquisa que permite a busca, a avaliação crítica e a síntese das evidências disponíveis do tema investigado. Inclui a análise de pesquisas relevantes no meio científico referente ao tema investigado, além de identificar lacunas do conhecimento que necessitam serem preenchidas por meio da realização de novas pesquisas ${ }^{15}$.

A construção da revisão integrativa segue seis etapas distintas, as quais foram utilizadas nesse estudo: 1. Identificação do tema ou questão de estudo; 2. Estabelecimento de critérios para inclusão e exclusão de estudos; 3. Definição das informações a serem extraídas dos estudos selecionados; 4. Análise crítica dos estudos; 5. Interpretação dos resultados obtidos com a revisão; 6 . Elaboração da síntese do conhecimento ${ }^{15}$.

Para guiar o estudo foi formulada a seguinte questão norteadora: Quais os fatores que influenciam a qualidade de vida do cuidador familiar da pessoa idosa com demência?

O levantamento bibliográfico foi realizado por meio da identificação e seleção dos estudos 
nas seguintes bases de dados: Base de Dados de Enfermagem (BDENF), Literatura Latino-Americana e do Caribe em Ciências da Saúde (Lilacs) e Medical Literature Analysis and Retrieval Sistem (Medline). Os estudos foram identificados mediante busca no site da Biblioteca Virtual em Saúde (BVS), que inclui publicações indexadas das bases de dados BDENF, Lilacs e Medline. Utilizou-se de forma pareada os descritores qualidade de vida, cuidadores, demência, doença de Alzheimer, doença por Corpos de Lewy, demência Frontotemporal e demência por múltiplos infartos, através do formulário avançado da base de dados. Por intermédio desse formulário é possível montar a "expressão de pesquisa" de maneira avançada, pois possibilita maior quantidade de campos para a busca na base de dados. Os descritores definidos e a quantidades de artigos identificados em cada base de dados estão apresentados na Tabela 1.

Os critérios de inclusão definidos para a seleção dos artigos foram: estudos primários desenvolvidos com seres humanos; artigos referentes à qualidade de vida do cuidador familiar da pessoa idosa com demência mensurada por instrumentos validados; publicações em português, inglês e espanhol na íntegra e indexadas nos referidos bancos de dados, no período de 2004 a dezembro de 2013. O período definido deve-se ao fato de os estudos da área da saúde terem uma evolução constante, sendo primordial a atualização.

A primeira etapa de seleção dos artigos consistiu na leitura exaustiva do título e resumo pelas pesquisadoras para a seleção de obras pertinentes ao objetivo deste estudo. Essa etapa permitiu se- lecionar quatro artigos do BDENF, 11 artigos da Lilacs e 45 artigos da Medline, totalizando 60 artigos. Ressalta-se que dois artigos apresentaram duplicidade nas bases de dados Lilacs e Medline. Após essa seleção todos os artigos selecionados foram submetidos à leitura na íntegra e analisados quanto aos critérios estabelecidos. Ao final, foram mantidos 11 artigos que compuseram a amostra final da presente revisão, sendo dois artigos da base de dados Lilacs, nove artigos da Medline e nenhum do BDENF.

A estratégia utilizada para a coleta de dados dos artigos foi à utilização de um instrumento construído e validado por Ursi ${ }^{16}$. Este instrumento contempla os seguintes itens: identificação do artigo, características metodológicas do estudo, avaliação do rigor metodológico, intervenções mensuradas e resultados encontrados. Para atender o objetivo do estudo, foram necessárias algumas adaptações do instrumento, devido ao fato dele ter sido originalmente proposto para a enfermagem perioperatória. As adaptações realizadas foram no item 3, cuja publicação em revista perioperatória foi substituída por publicação em revista de enfermagem de outras especialidades, e o item 4, subitem 3 , que constava diagnóstico e tipo de cirurgia, foi excluído.

Os estudos selecionados que compuseram a amostra foram classificados em níveis, conforme proposto por Stetler et al. ${ }^{17}$ : nível I - estudos de metanálise de múltiplos estudos controlados; nível II - estudo experimental individual/ensaio clínicos randomizados; nível III - estudos quase experimentais como ensaio clínico não randomizado, controlado e não aleatorizado - grupo pré

Tabela 1. Quantitativo de artigos identificados de acordo com os descritores definidos nas bases de dados - Belo Horizonte, 2014.

\begin{tabular}{|c|c|c|}
\hline $\begin{array}{c}\text { Base } \\
\text { de dados }\end{array}$ & $\begin{array}{l}\text { Descritores cruzados } \\
\text { concomitantemente }\end{array}$ & $\begin{array}{l}\text { Número } \\
\text { de artigos } \\
\text { identificados }\end{array}$ \\
\hline LILACS & $\begin{array}{l}\text { Qualidade de Vida/ cuidadores /demência /Doença de Alzheimer/ /Doença } \\
\text { por Corpos de Lewy/Demência Frontotemporal/Demência por Múltiplos } \\
\text { Infartos }\end{array}$ & 24 \\
\hline MEDLINE & $\begin{array}{l}\text { Qualidade de Vida/ cuidadores /demência /Doença de Alzheimer/ /Doença } \\
\text { por Corpos de Lewy/Demência Frontotemporal/Demência por Múltiplos } \\
\text { Infartos }\end{array}$ & 438 \\
\hline BDENF & $\begin{array}{l}\text { Qualidade de Vida/ cuidadores /demência /Doença de Alzheimer/ /Doença } \\
\text { por Corpos de Lewy/Demência Frontotemporal/Demência por Múltiplos } \\
\text { Infartos }\end{array}$ & 15 \\
\hline Total & & 477 \\
\hline
\end{tabular}


e pós-teste; nível IV - estudo não experimental, como pesquisa correlacional descritiva e qualitativa, ou estudo de caso. Não foram incluídos nessa revisão artigos de nível V, ou seja, relatórios de casos ou dados obtidos sistematicamente; e de nível VI, que incluem opinião de especialistas ou relato de experiência.

A análise dos resultados dos dados ocorreu de forma descritiva, permitindo avaliar as seguintes características de cada artigo: identificação da publicação, critérios de avaliação de QV e metodologia dos estudos.

\section{Resultados}

Foram analisados 11 artigos na íntegra, sendo três estudos experimentais individuais (ensaio clínico randomizado), dois estudos quase experimentais e seis estudos quantitativos.

A maior parte dos estudos foi encontrada em periódicos internacionais $(n=9)$. Em relação ao idioma, oito eram em inglês, dois em português e um em espanhol. No que se refere ao País sede do estudo, foram desenvolvidos dois estudos no Brasil. Em cada um dos seguintes países foi desenvolvido um estudo: Canadá, China, Espanha, Estados Unidos, Itália, Japão, Países Baixos, Taiwan e Turquia. Quanto ao periódico de publicação, foram identificados dez periódicos distintos, sendo seis revistas específicas de Gerontologia e/ou Geriatria, três de Saúde Pública e um artigo em uma revista da área da enfermagem/medicina.

No que se refere à caracterização dos estudos, quanto ao ano de publicação em 2011e 2006 , foram publicados três artigos; no ano de 2012 foram publicados dois artigos; e nos anos 2013 2010, 2009, 2008, 2005 e 2004, um artigo a cada ano. Quanto à formação profissional dos autores responsáveis, os artigos foram publicados por enfermeiros, médicos (psiquiatras, neurologistas e geriatras), estatísticos, neurocientistas, psicólogos e farmacêutica.

De acordo com o delineamento da pesquisa e o nível de evidência avaliado, houve predomínio de estudos descritivos nível IV (estudos descritivos) correspondendo a seis artigos; três artigos referiam ao nível III (estudos experimentais individuais) e dois estudos de nível II (estudo casocontrole ) esse nível considerado como evidência forte.

Quanto ao método utilizado para a avaliação de QV, foram utilizados seis instrumentos diferentes para mensurar a QV do cuidador. O Qua- dro 1 apresenta o instrumento utilizado em cada estudo: Os resultados obtidos são visualizados no Quadro 2 que se segue, onde através de sinopse são identificada(o)s autora(e)s, ano de publicação, país, objetivo, delineamento do estudo, nível de evidência, amostra e resultado do estudo.

\section{Discussão}

Analisando o quantitativo e o desenho dos estudos, percebe-se que a qualidade de vida do cuidador familiar do idoso com demência é um tema ainda pouco abordado na literatura, principalmente em nível nacional. Os estudos que propõem intervenções e analisam sua efetividade são insuficientes ao nível internacional e escassos de âmbito nacional, assim como a elaboração de estratégias que possibilitem a melhora na QV e a diminuição da sobrecarga do cuidador.

O perfil de cuidadores familiares de pessoas idosas encontrados nos estudos corrobora com dados encontrados na literatura. A maioria dos cuidadores são do sexo feminino, cônjuges e filhas ${ }^{18-20}$. Contudo, destaca-se que há crescente participação dos homens e esse dado foi encontrado em alguns estudos ${ }^{21-23}$.

Atualmente, o número de cuidadores idosos cuidando de um idoso é um dado relevante. Um dos estudos encontrou alto percentual da amostra $(64 \%)$ de familiares com 60 anos ou mais a exercerem o papel de cuidador ${ }^{22}$. Tal dado corrobora com outros estudos que apontam que esse número é crescente $\mathrm{e}^{5,18}$.

Com o aumento da longevidade, há uma tendência de crescimento no número de cuidadores com idade superior a 60 anos, esses na maioria das vezes cuidam de seus cônjuges ou progenitores $^{24}$.

Nesse contexto é essencial considerar as condições físicas desses cuidadores que podem se tornar doentes em potencial, além disso, se faz necessário refletir que a sua capacidade funcional está constantemente em risco ${ }^{18}$.

A partir da análise dos artigos, identificou-se que os fatores que influenciam a QV do cuidador familiar podem ser agrupados nos seguintes aspectos: depressão; má qualidade do sono; tipo de demência e sintomas neuropsiquiátricos; apoio, suporte social e acesso aos serviços de saúde; lazer; problemas de saúde pré-existentes; intervenções subsidiadas com treinamento para o cuidador e espiritualidade. 
Quadro 1. Instrumentos de QV usados nos artigos analisados. Belo Horizonte, 2014.

\begin{tabular}{|c|c|c|}
\hline Instrumentos & Funções e itens de avaliação & Estudos (autoria/ano/país) \\
\hline $\begin{array}{l}\text { Short Form Health } \\
\text { Survey-36 (SF-36) }\end{array}$ & $\begin{array}{l}\text { Avalia QV geral e os seguintes domínios: } \\
\text { capacidade funcional, aspectos físicos, } \\
\text { dor, estado geral de saúde, vitalidade, } \\
\text { aspectos sociais, aspectos emocionais e } \\
\text { saúde mental. }\end{array}$ & $\begin{array}{l}\text { Kuo LM, Huang HL,Huang HL, Liang J, } \\
\text { Chiu YC, Chen ST et al. 2013; Taiwan }{ }^{21} \\
\text { Bormann J, Kathleen A, Warren MS, } \\
\text { Regalbuto LCSW, Glaser D, Kelly AMSN et } \\
\text { al. 2009; E.U.A. }{ }^{25} \\
\text { Riedijk SR, Vugt ME, Duivenvoorden HJ, } \\
\text { Niermeijer MF, Swieten VJC, Verhey FRJ et } \\
\text { al. 2006; Países Baixos }{ }^{26}\end{array}$ \\
\hline $\begin{array}{l}\text { World Health } \\
\text { Organization } \\
\text { Quality of Life-bref } \\
\text { (WHOQOL-bref) } \\
\text { MCGILL QV } \\
\text { questionnaire }\end{array}$ & $\begin{array}{l}\text { Avalia QV geral e os seguintes domínios: } \\
\text { saúde física, mental ou emocional, social, } \\
\text { funcional ou de desempenho de papel }\end{array}$ & $\begin{array}{l}\text { Chien WT, Lee YM. 2008; China }{ }^{23} \\
\text { Takai M, Takahashi M, Iwamitsu Y, Oishi S, } \\
\text { Miyaoka H. 2011; Japão }{ }^{27} \\
\text { Cupidi C, Realmuto S, Lo Coco G, Cinturino } \\
\text { A, Talamanca S, Arnao V et al. 2012; Itália }{ }^{28}\end{array}$ \\
\hline CQdV-DA & $\begin{array}{l}\text { Avalia QV geral e os seguintes domínios: } \\
\text { saúde física, disposição, humor, moradia, } \\
\text { memória, família, casamento, amigos, } \\
\text { você em geral, capacidade de fazer } \\
\text { tarefas, capacidade de fazer atividades de } \\
\text { lazer e dinheiro. }\end{array}$ & $\begin{array}{l}\text { Keika I, Pedrazzani ES, Pavarini SCI. 2010; } \\
\text { Brasil22 }^{22} \\
\text { Bartfay E, Bartfay W. 2011; Canadá29 } \\
\text { Borghi AC, Sassá AH, Matos PCB, Decesaro } \\
\text { MN, Marcon SS. 2011; Brasil }{ }^{30}\end{array}$ \\
\hline Escala DUKE & $\begin{array}{c}\text { Avalia QV geral e os seguintes domínios: } \\
\text { saúde física, mental, social, percebida e } \\
\text { autoestima. }\end{array}$ & $\begin{array}{l}\text { Kuzu N, Beser N, Zencir M, Sahiner T, } \\
\text { ahiner, MD, Nesrin E et al., 2005; Turquia }\end{array}$ \\
\hline Euro QoL-5D & $\begin{array}{c}\text { Avalia QV geral e os seguintes domínios: } \\
\text { mobilidade, cuidados próprios, atividade } \\
\text { habitual, dor/desconforto e ansiedade/ } \\
\text { depressão. }\end{array}$ & $\begin{array}{l}\text { Llacha BN. Suriñachb L, Gamisansb MR et } \\
\text { al. 2004; Espanha }\end{array}$ \\
\hline
\end{tabular}

\section{- Depressão}

Nesta temática, alguns estudos analisados destacam que os sintomas psiquiátricos dos pacientes, tais como delírio, ansiedade e depressão e comportamentos agressivos aumentam a carga sobre os cuidadores familiares, o que interfere na saúde mental e QV $21,26,30$. Outro estudo ${ }^{31}$ apontou que a alta prevalência de problemas de saúde entre cuidadores reflete negativamente em sua QV. Os autores desse estudo encontraram dados preocupantes, ao afirmarem que $84 \%$ dos cuidadores relataram ter problemas físicos e $94 \%$, problemas psicológicos. De acordo com a avaliação da QV nessa amostra, os cuidadores apresentaram problemas nas dimensões de mobilidade, atividades usuais, dor ou desconforto, ansiedade ou depressão do que a população em geral.
Um dos artigos analisados identificou que as mulheres apresentam mais quadros de depressão ${ }^{27}$. Um estudo assinala que essa realidade pode estar associada ao grande número de papéis que a mulher desempenha na sociedade ${ }^{33}$.

Estudos destacam que a depressão do cuidador é frequentemente associada com a depressão do paciente ${ }^{26}$. As situações de estresse crônico vivenciada pelos familiares, o impacto psicológico que o cuidado prolongado gera e a própria característica da doença que é progressiva são temáticas enfatizadas em alguns estudos como um fator de risco para depressão $0^{21,28,33}$.

- Má qualidade do sono

Nesta temática, um dos estudos da amostra confirma a alta prevalência de má qualidade do sono em cuidadores de pacientes idosos com 
Quadro 2. Sinopse dos estudos realizados com cuidadores familiares de idosos com demência. Belo Horizonte, 2014.

\begin{tabular}{|c|c|c|c|c|}
\hline Autores & Objetivo & $\begin{array}{l}\text { Delineamento/ } \\
\text { Nível de evidência }\end{array}$ & Amostra & Resultado \\
\hline $\begin{array}{l}\text { Kuo LM, Huang } \\
\text { HL, Huang HL, } \\
\text { Liang J, Chiu YC, } \\
\text { Chen ST. et al. } \\
\text { 2013; } \\
\text { Taiwan }^{21}\end{array}$ & $\begin{array}{l}\text { Examinar os efeitos } \\
\text { de um programa de } \\
\text { treinamento na QV e } \\
\text { sintomas depressivos } \\
\text { em cuidadores } \\
\text { familiares de idosos } \\
\text { com demência. }\end{array}$ & $\begin{array}{l}\text { Estudo } \\
\text { Experimental } \\
\text { individual } \\
\text { (ensaio clínico } \\
\text { randomizado) } \\
\text { Nível II }\end{array}$ & $\begin{array}{l}\mathrm{n}=63 \text { idosos com } \\
\text { demência e seus } \\
\text { respectivos cuidadores } \\
\mathrm{n}=66 \text { idosos com } \\
\text { demência e seus } \\
\text { respectivos cuidadores } \\
\text { (grupo controle) }\end{array}$ & $\begin{array}{l}\text { Cuidadores familiares do grupo } \\
\text { de intervenção tiveram melhores } \\
\text { resultados na saúde relacionada } \\
\text { à dor corporal. Notou-se menor } \\
\text { índice de depressão comparado aos } \\
\text { do grupo controle. }\end{array}$ \\
\hline $\begin{array}{l}\text { Chien WT, Lee } \\
\text { YM. 2008; } \\
\text { China }^{23}\end{array}$ & $\begin{array}{l}\text { Testar a eficácia de } \\
\text { um programa de } \\
\text { gestão para famílias } \\
\text { chinesas de idosos } \\
\text { com demência. }\end{array}$ & $\begin{array}{l}\text { Estudo } \\
\text { Experimental } \\
\text { individual } \\
\text { (ensaio clínico } \\
\text { randomizado) } \\
\text { Nível II }\end{array}$ & $\begin{array}{l}\mathrm{n}=44 \text { grupo } \\
\text { intervenção } \\
\text { composto por idosos } \\
\text { com demência e } \\
\text { cuidadores familiares; } \\
\mathrm{n}=44 \text { Grupo controle } \\
\text { composto por idosos } \\
\text { com demência e } \\
\text { cuidadores familiares. }\end{array}$ & $\begin{array}{l}\text { Os resultados do estudo sugerem } \\
\text { que o fornecimento de um } \\
\text { programa de gestão de cuidados } \\
\text { multiprofissional com enfoque } \\
\text { na sensibilidade cultural podem } \\
\text { melhorar os sintomas dos } \\
\text { idosos com demência, sintomas } \\
\text { psicossociais dos cuidadores } \\
\text { familiares e pode reduzir as taxas } \\
\text { de pacientes institucionalizados. }\end{array}$ \\
\hline $\begin{array}{l}\text { Bormann J, } \\
\text { Kathleen A, } \\
\text { Warren MS, } \\
\text { Regalbuto } \\
\text { LCSW,Glaser D, } \\
\text { Kelly AMSN et al. } \\
\text { 2009; } \\
\text { E.U.A. }^{25}\end{array}$ & $\begin{array}{l}\text { Avaliar a viabilidade e } \\
\text { eficácia da intervenção } \\
\text { com mantras através } \\
\text { de chamadas de } \\
\text { teleconferência, } \\
\text { com base na aflição } \\
\text { psicológica e QV } \\
\text { em uma amostra de } \\
\text { cuidadores familiares } \\
\text { de idosos com } \\
\text { demência. }\end{array}$ & $\begin{array}{l}\text { Estudo } \\
\text { Experimental } \\
\text { individual } \\
\text { (ensaio clínico } \\
\text { randomizado) } \\
\text { Nível II }\end{array}$ & $\begin{array}{l}\mathrm{n}=21 \text {, porém } 16 \\
\text { completaram a } \\
\text { intervenção (período } \\
\text { de intervenção: } 36 \\
\text { semanas) }\end{array}$ & $\begin{array}{l}\text { A sobrecarga do cuidador familiar } \\
\text { nesta amostra foi considerada alta, } \\
\text { houve diminuição na } 8^{\text {a }} \text { semana } \\
\text { de intervenção. O programa } \\
\text { possibilitou reduções significativas } \\
\text { na maioria das medidas de } \\
\text { sofrimento psíquico do cuidador e } \\
\text { houve melhoria na QV. }\end{array}$ \\
\hline $\begin{array}{l}\text { Cupidi C, } \\
\text { Realmuto S, Lo } \\
\text { Coco G, Cinturino } \\
\text { A, Talamanca S, } \\
\text { Arnao V et al. } \\
2012 . \\
\text { Itália }^{28}\end{array}$ & $\begin{array}{l}\text { Investigar a relação } \\
\text { entre auto relato de } \\
\text { falta de sono e QV de } \\
\text { cuidadores familiares. }\end{array}$ & $\begin{array}{l}\text { Estudo quase } \\
\text { experimental } \\
\text { (caso controle) } \\
\text { Nível III }\end{array}$ & $\begin{array}{l}\mathrm{n}=40 \text { idosos } \\
\text { com Doença de } \\
\text { Alzheimer (DA) } \\
\text { e seus respectivos } \\
\text { cuidadores; } \\
\mathrm{n}=40 \text { idosos com } \\
\text { Doença de Parkinson } \\
\text { (DP) sem demência } \\
\text { e seus cuidadores } \\
\text { principais. } \\
\mathrm{n}=150 \text { idosos e seus } \\
\text { respectivos cuidadores } \\
\text { (grupo controle) }\end{array}$ & $\begin{array}{l}\text { Cuidadores de idosos com DA } \\
\text { relataram maior fardo, pior QV e } \\
\text { bem-estar, tiveram escores mais } \\
\text { elevados no índice de depressão } \\
\text { comparado aos cuidadores } \\
\text { de pacientes com DP. Os } \\
\text { cuidadores que relataram insônia } \\
\text { apresentaram mais sintomas } \\
\text { depressivos. O estudo identificou } \\
\text { que quanto pior a qualidade do } \\
\text { sono também pior a QV percebida } \\
\text { pelo cuidador. }\end{array}$ \\
\hline
\end{tabular}

diagnóstico de DA e $\mathrm{DP}^{28}$. Evidenciaram-se escores elevados para depressão através do instrumento Beck Depression Inventory (BDI) em cuidadores de idosos com DA, quando comparado com cuidadores de pacientes com DP. Além disso, os cuidadores que apresentaram insônia eram significativamente mais sobrecarregados e apresentavam mais sintomas depressivos. Concluiuse que os índices de QV em geral, bem como a satisfação com muitos domínios específicos da vida, foram correlacionados com a qualidade de sono. Portanto, qualidade do sono insatisfatória 
Quadro 2. continuação

\begin{tabular}{|c|c|c|c|c|}
\hline Autores & Objetivo & $\begin{array}{c}\text { Delineamento/ } \\
\text { Nível de evidência }\end{array}$ & Amostra & Resultado \\
\hline $\begin{array}{l}\text { Keika I, } \\
\text { Pedrazzani ES, } \\
\text { Pavarini SCI. } \\
2010 ; \\
\text { Brasil }^{22}\end{array}$ & $\begin{array}{l}\text { Comparar a } \\
\text { percepção geral e de } \\
\text { cada dimensão de } \\
\text { QV, de um grupo } \\
\text { de cuidadores de } \\
\text { idosos com DA com } \\
\text { um grupo de não } \\
\text { cuidadores }\end{array}$ & $\begin{array}{l}\text { Estudo quase } \\
\text { experimental } \\
\text { (caso controle) } \\
\text { Nível III }\end{array}$ & $\begin{array}{l}\mathrm{n}=53 \text { cuidadores } \\
\text { familiares de idosos } \\
\text { com DA; } \\
\mathrm{n}=53 \text { grupo controle } \\
\text { de não cuidadores. }\end{array}$ & $\begin{array}{l}\text { Os familiares encontravam } \\
\text { conforto e amparo na religião } \\
23 \% \% \text {. Na amostra, apenas } 6 \% \\
\text { dos indivíduos haviam sido } \\
\text { capacitados para serem cuidadores; } \\
4 \% \text { estavam em tratamento } \\
\text { psicológico; } 4 \% \text { tinham hobbies; } \\
2 \% \text { frequentavam a Universidade } \\
\text { Aberta à Terceira Idade; } 26 \% \\
\text { disseram não dispor de qualquer } \\
\text { auxílio, apoio ou oportunidades } \\
\text { de lazer. Os itens de qualidade } \\
\text { de vida que apresentavam } \\
\text { razão desfavorável para o grupo } \\
\text { cuidadores foram: saúde física, } \\
\text { disposição, humor, memória, você } \\
\text { em geral e capacidade para fazer } \\
\text { atividades de lazer. }\end{array}$ \\
\hline $\begin{array}{l}\text { Bartfay E, Bartfay } \\
\text { W. 2011; } \\
\text { Canadá }^{29}\end{array}$ & $\begin{array}{l}\text { Determinar como } \\
\text { intervenções de base } \\
\text { comunitárias para } \\
\text { o cuidador familiar } \\
\text { afetam a QV de idosos } \\
\text { com DA. }\end{array}$ & $\begin{array}{l}\text { Estudo descritivo } \\
\text { (abordagem } \\
\text { quantitativa) } \\
\text { Nível IV }\end{array}$ & $\begin{array}{l}\mathrm{n}=28 \text { Grupo } 1 \text { - } \\
\text { cuidadores familiares } \\
\text { de idosos com DA que } \\
\text { participaram de grupo } \\
\text { de apoio; } \\
\mathrm{n}=15 \text { Grupo 2- } \\
\text { cuidadores familiares } \\
\text { de idosos com DA } \\
\text { que eram clientes do } \\
\text { programa dia adulto; } \\
\mathrm{n}=19 \text { Grupo 3- } \\
\text { Cuidadores } \\
\text { familiares de idosos } \\
\text { que participam do } \\
\text { programa dia adulto. }\end{array}$ & $\begin{array}{l}\text { QV dos cuidadores familiares de } \\
\text { idosos com DA foi semelhante ao } \\
\text { dos cuidadores de idosos sem DA. } \\
\text { Sendo assim, os resultados podem } \\
\text { sugerir que esses programas } \\
\text { fornecem benefícios indiretos aos } \\
\text { cuidadores. }\end{array}$ \\
\hline $\begin{array}{l}\text { Borghi AC, Sassá } \\
\text { AH, Matos PCB, } \\
\text { Decesaro MN, } \\
\text { Marcon SS. 2011; } \\
\text { Brasil }^{30}\end{array}$ & $\begin{array}{l}\text { Caracterizar o perfil } \\
\text { dos cuidadores } \\
\text { e os idosos } \\
\text { segundo variáveis } \\
\text { sociodemográficas. }\end{array}$ & $\begin{array}{l}\text { Estudo descritivo } \\
\text { (abordagem } \\
\text { quantitativa) } \\
\text { Nível IV }\end{array}$ & $\begin{array}{l}\mathrm{n}=50 \text { cuidadores } \\
\text { familiares de idosos } \\
\text { com DA }\end{array}$ & $\begin{array}{l}\text { Os cuidadores consideraram } \\
\text { positivos doze itens da escala } \\
\text { QV dos idosos, destacando-se } \\
\text { moradia }(98 \%) \text {, você em geral e } \\
\text { a vida em geral ( } 86 \%) \text {. A única } \\
\text { avaliação negativa foi referente } \\
\text { à capacidade para atividades de } \\
\text { lazer onde } 58 \% \text { dos cuidadores se } \\
\text { mostraram insatisfeitos. Este dado } \\
\text { possivelmente está relacionado à } \\
\text { sobrecarga que limita o cotidiano } \\
\text { do familiar e altera a rotina que } \\
\text { antes incluía programas de lazer. }\end{array}$ \\
\hline
\end{tabular}

está diretamente relacionada a baixos escores de QV. A literatura tem destacado a associação en- tre transtornos do sono com a depressão. Uma parcela significativa dos pacientes depressivos 


\begin{tabular}{|c|c|c|c|c|}
\hline \multirow{4}{*}{ 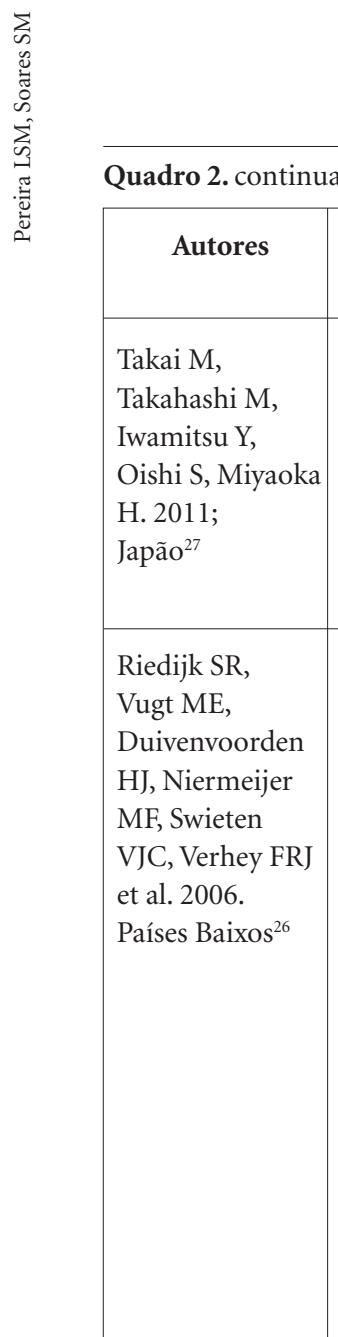 } & ação & & & \\
\hline & Objetivo & $\begin{array}{l}\text { Delineamento/ } \\
\text { Nível de evidência }\end{array}$ & Amostra & Resultado \\
\hline & $\begin{array}{l}\text { Identificar a relação } \\
\text { entre a QV do cuidador } \\
\text { e do } \\
\text { idoso com DA. }\end{array}$ & $\begin{array}{l}\text { Estudo descritivo } \\
\text { (abordagem } \\
\text { quantitativa) } \\
\text { Nível IV }\end{array}$ & $\begin{array}{l}\mathrm{N}=118 \text { cuidadores } \\
\text { familiares de idosos } \\
\text { com demência }\end{array}$ & $\begin{array}{l}\text { A análise revelou que depressão e } \\
\text { sexo feminino estiveram associados } \\
\text { com pior escore de QV. Os } \\
\text { cuidadores familiares têm longo e } \\
\text { profundo contato interpessoal com } \\
\text { os idosos, o que pode induzir efeitos } \\
\text { psicológicos negativos. }\end{array}$ \\
\hline & $\begin{array}{l}\text { Examinar os fatores } \\
\text { preditivos de QV do } \\
\text { cuidadores familiares de } \\
\text { idosos com demência. }\end{array}$ & $\begin{array}{l}\text { Estudo descritivo } \\
\text { (abordagem } \\
\text { quantitativa) } \\
\text { Nível IV }\end{array}$ & $\begin{array}{l}\mathrm{n}=90 \text { cuidadores } \\
\text { familiares de idosos } \\
\text { com DA; } \\
\mathrm{N}=63 \text { cuidadores } \\
\text { familiares de idosos } \\
\text { com FTD. }\end{array}$ & $\begin{array}{l}\text { Idosos com FTD e idosos com } \\
\text { DA diferiram significativamente } \\
\text { na ocorrência de sintomas } \\
\text { neuropsiquiátricos, pacientes com } \\
\text { FTD sofriam de euforia, apatia, } \\
\text { desinibição e comportamento motor } \\
\text { aberrante, enquanto pacientes } \\
\text { com DA sofriam de depressão. Os } \\
\text { cuidadores familiares de idosos } \\
\text { das respectivas doenças não } \\
\text { diferiram significativamente da } \\
\text { carga emocional. Entretanto, os } \\
\text { cuidadores de idosos com FTD } \\
\text { sentiram significativamente mais } \\
\text { sobrecarregados e com maiores } \\
\text { dificuldades de encontrar suporte nos } \\
\text { serviços de saúde comparados aos } \\
\text { cuidadores de idosos com DA. }\end{array}$ \\
\hline $\begin{array}{l}\text { Kuzu N, Beser } \\
\text { N, Zencir M, } \\
\text { Sahiner T, } \\
\text { ahiner, MD, } \\
\text { Nesrin E et al. } \\
\text { 2005; } \\
\text { Turquia }^{31}\end{array}$ & $\begin{array}{l}\text { Diferenciar sobrecarga } \\
\text { do cuidador familiar do } \\
\text { idoso com FTD da carga } \\
\text { do cuidador familiar do } \\
\text { idoso com DA. Avaliar a } \\
\text { sobrecarga e QV. }\end{array}$ & $\begin{array}{l}\text { Estudo descritivo } \\
\text { (abordagem } \\
\text { quantitativa) } \\
\text { Nível IV }\end{array}$ & $\begin{array}{l}\mathrm{n}=32 \text { cuidadores } \\
\text { familiares, } \\
\mathrm{n}=32 \text { idosos com } \\
\mathrm{DA}\end{array}$ & $\begin{array}{l}\text { Este estudo mostrou que o Programa } \\
\text { reduziu os problemas de idosos com } \\
\text { DA e seus cuidadores familiares, além } \\
\text { de proporcionar aumento da QV, } \\
\text { diminuição da ansiedade e depressão. } \\
\text { Além disso, foi demonstrado } \\
\text { melhora do conhecimento do } \\
\text { cuidador em relação ao tipo de } \\
\text { demência, diminuição do fardo } \\
\text { familiar, melhora nas habilidades } \\
\text { de enfrentamento e retardo na } \\
\text { institucionalização. }\end{array}$ \\
\hline $\begin{array}{l}\text { Llacha BN. } \\
\text { Suriñachb L, } \\
\text { Gamisansb MR } \\
\text { et al. 2004; } \\
\text { Espanha }^{32}\end{array}$ & $\begin{array}{l}\text { Investigar a eficácia } \\
\text { de um Programa de } \\
\text { Educação Integral } \\
\text { Individualizado } \\
\text { sobre os problemas } \\
\text { susceptíveis de serem } \\
\text { experimentados por } \\
\text { cuidadores familiares de } \\
\text { idosos com DA } \\
\text { Avaliar o impacto na QV } \\
\text { e sobrecarga percebida } \\
\text { por cuidadores } \\
\text { familiares de idosos com } \\
\text { DA na Espanha. }\end{array}$ & $\begin{array}{l}\text { Estudo descritivo } \\
\text { (abordagem } \\
\text { quantitativa) } \\
\text { Nível IV }\end{array}$ & $\begin{array}{l}\mathrm{n}=268 \text { idosos com } \\
\mathrm{DA} \\
\mathrm{n}=268 \text { cuidadores } \\
\text { familiares }\end{array}$ & $\begin{array}{l}\text { A alta prevalência de problemas } \\
\text { de saúde entre cuidadores reflete } \\
\text { em sua QV, } 84 \% \text { dos cuidadores } \\
\text { relataram ter problemas físicos e } 94 \% \\
\text { problemas psicológicos. } \\
\text { Os cuidadores apresentaram } \\
\text { problemas nas dimensões de } \\
\text { mobilidade, atividades usuais, } \\
\text { dor ou desconforto, ansiedade ou } \\
\text { depressão do que a população em } \\
\text { geral. Tal resultado reflete na própria } \\
\text { percepção do cuidador que atribuiu } \\
\text { uma pior avaliação de sua saúde geral } \\
\text { comparada à população em geral. }\end{array}$ \\
\hline
\end{tabular}


se queixa da deterioração tanto da quantidade como da qualidade do sono. Portanto, além dos estudos sobre o sono, que têm apresentado essa associação, torna-se necessário a investigação da depressão, estratégias para o diagnóstico, tratamento e, inclusive, a identificação de indivíduos suscetíveis ao seu desenvolvimento ${ }^{33}$.

\section{- Tipo de Demência}

\section{e sintomas neuropsiquiátricos}

Nota-se que a demência mais estudada foi à Doença de Alzheimer, infere-se nesse resultado o fato da DA se destacar e ser a mais prevalente entre as demências, representando 50 a 70\% dos $\operatorname{casos}^{34}$. Dos onze estudos, três explicitaram outras demências, sendo que dois abordaram DA e FTD, e um, DA, demência mista e DCL, concomitante. Os outros estudos estudaram DA ou não deixaram claro com qual tipo de demência o paciente foi diagnosticado. Ressalta-se que um estudo analisou o cuidador familiar que cuida de paciente com DA e o que cuida de paciente com Doença de Parkinson (DP) sem demência.

O estudo que avaliou pacientes FTD e pacientes com DA indicou que houve diferença significativa quanto à ocorrência de sintomas neuropsiquiátricos. Pacientes FTD eram mais propensos a sintomas como euforia, apatia, desinibição e comportamento motor aberrante, enquanto pacientes com DA eram mais deprimidos. Cuidadores de pacientes idosos com FTD e cuidadores de pacientes idosos com DA não diferiram significativamente da carga emocional que experimentaram devido a perturbações neuropsiquiátricas dos pacientes. Os cuidadores de pacientes idosos com FTD sentiram-se significativamente mais sobrecarregados pelo processo de cuidar do que os cuidadores de pacientes idosos com $\mathrm{DA}^{26}$.

Entretanto, outro estudo concluiu que cuidadores de pacientes idosos com DA relataram maior fardo, menor QV, bem-estar existencial e domínios de apoio e Beck Depression Inventory (BDI) mais elevados do que cuidadores de pacientes idosos com DP. No grupo DA, os cuidadores que apresentaram insônia eram significativamente mais sobrecarregados e apresentavam mais sintomas depressivos ${ }^{28}$.

O tipo de demência é um fator que influencia a QV do cuidador. Entretanto, destaca-se que estudos indicam que, de acordo com os estágios do processo demencial, os próprios sintomas da doença geram diversas demandas, isso torna a tarefa do cuidado cada vez mais complexa ${ }^{35}$.

Ressalta-se que apenas um estudo ${ }^{21}$ especificou a gravidade da doença dos pacientes (demência leve, moderada e grave), entretanto não foi avaliado a QV e os fatores relacionados dos seus respectivos cuidadores de forma distinta.

- Apoio, suporte social e acesso

aos serviços de saúde

Alguns dos trabalhos analisados versam sobre o apoio, suporte social e o acesso aos serviços de saúde. Ressaltam a sobrecarga proveniente do cuidado e a necessidade de apoio ${ }^{21,22,25,28-31}$.

Em estudo que teve como método uma intervenção de base comunitária envolvendo pacientes com DA e grupo-controle, bem como seus cuidadores familiares, constatou que a QV de ambos os cuidadores foi semelhante após o programa de intervenção comunitária ${ }^{29}$.

Outro estudo quantitativo com a participação de 53 cuidadores confirma a piora da QV dos cuidadores que receberam auxílio de apenas uma pessoa para dividir a tarefa de cuidar em fins de semana e eventuais necessidades. Nessa amostra estudada, 26\% disseram não dispor de qualquer auxílio, apoio ou oportunidades de lazer. $\mathrm{Na}$ avaliação de QV os itens que apresentavam razão desfavorável para o grupo de cuidadores foram seis: saúde física, disposição, humor, memória, você em geral e capacidade para fazer atividades de lazer ${ }^{22}$.

Devido ao fato da DA ser a demência mais prevalente, um dos estudos destaca que os cuidadores FTD têm mais dificuldade em encontrar suporte no sistema de saúde ${ }^{26}$.

A família, e principalmente o cuidador, tem necessidades tanto nos aspectos materiais quanto emocionais, por isso é essencial um gerenciamento da atenção do idoso que conecte os familiares, a comunidade e os serviços de apoio, possibilitando a integração e criação de estratégias que favoreçam no planejamento da atenção, contribuindo com a qualidade de vida dos seus cuidadores ${ }^{13,36}$.

Um estudo que trata sobre os aspectos do desenvolvimento de políticas de atenção ao idoso no Brasil afirma que o Estado se apresenta com responsabilidades reduzidas, as maiores das atribuições e responsabilidades acerca do cuidado são delegadas à família. Atualmente, inexistem políticas referentes aos papéis atribuídos às famílias e cuidadores, como consequência, o apoio e a rede de suporte ao idoso ocorrem de maneira informal ${ }^{37}$.

\section{- Lazer}

Em relação ao lazer, em um dos estudos analisado, a única avaliação negativa acerca da QV dos cuidadores familiares foi referente à capacidade para atividades de lazer. A amostra foi composta de 50 cuidadores, sendo que 58\% dos 
entrevistados se mostraram insatisfeitos. Os autores sugerem que esse dado possivelmente esteja relacionado à sobrecarga que é um fator limitador no cotidiano do familiar e altera a rotina que antes incluía programas de lazer ${ }^{30}$.

Um estudo quantitativo com amostra $\mathrm{n}=53$, apenas $2(4 \%)$ dos cuidadores tinham hobbies e $1(2 \%)$ frequentava a Universidade Aberta à Terceira Idade I $^{22}$.

A literatura aponta que ser cuidador no ambiente domiciliar, implica em descuidar da sua vida pessoal e muitas vezes profissional. $\mathrm{O}$ cuidador, neste momento, tem como prioridade cuidar de seu familiar e isso implica em mudanças no contexto de vida para atender as necessidades do idoso $^{13,38}$.

- Problemas de saúde pré-existentes

Um dos estudos analisados identificou que a alta prevalência de problemas de saúde entre cuidadores também reflete em sua QV. Na amostra de 268 cuidadores, $84 \%$ relataram ter problemas físicos e $94 \%$, problemas psicológicos. Isso também se reflete na valorização atribuída à saúde geral, cuja amostra também marcou pior avaliação de QV ${ }^{31}$.

Estudos na literatura mostram que prestar cuidado a uma pessoa idosa com alterações cognitivas pode acarretar sobrecarga física e emocional à vida do cuidador. A perda da capacidade do idoso em executar as AVD's tornando-se dependente é um fato que demanda do cuidador um cuidado físico mais intenso e atenção ${ }^{10,13,14,39}$.

Um estudo destaca que a grande maioria dos cuidadores ainda tem informações insuficientes e faltam suportes necessários para prestar assistência necessária a seu familiar. Na amostra estudada $(\mathrm{n}=53)$, apenas $6 \%$ dos indivíduos haviam sido capacitados para serem cuidadores ${ }^{28}$. Dessa forma essa tarefa se torna muitas vezes um risco de desgaste físico, emocional e social, pode acarretar doenças como depressão, ansiedade, alterações do sono, e inclusive progressão de doenças pré - existentes ${ }^{40,41}$.

\section{- Intervenções subsidiadas}

\section{com treinamento para o cuidador}

Os estudos analisados relatam que há evidências de que o bem-estar psicológico dos cuidadores, depressão, e os resultados sociais melhoraram após intervenções do grupo de apoio, incluindo o apoio mútuo e educação ${ }^{21,29}$. O cuidar de um membro da família com demência traz efeitos adversos à saúde psicossocial, portanto intervenções com enfoque na melhoria da saúde mental acarreta benefícios para os cuidadores familiares e possibilita um retardo na institucionalização ${ }^{23}$.
Um dos estudos pontua ${ }^{29}$ que foi identificado benefício através de uma intervenção com intuito de melhorar a QV do cuidador, porém sugere que o pequeno tamanho da amostra requer uma interpretação dos resultados com cautela, o que de fato é coerente.

O programa de intervenção foi clinicamente eficiente na melhoria da dor física dos cuidadores, vitalidade, percepção geral da saúde e função social, do que para outros resultados relacionados à saúde ${ }^{21}$. O resultado da intervenção com múltiplos componentes estruturados e adaptados para perfis de risco individuais pode aumentar a QV de diversos cuidadores de pessoas idosas com demência ${ }^{23}$.

Estudo na área da Enfermagem Gerontológica mostra que há uma carência de capacitação e suporte tanto para os profissionais quanto para os cuidadores familiares/leigos ${ }^{42}$.

Dados da literatura indicam que as intervenções em cuidadores têm surgido nos últimos 15 anos, contudo há carência sobre essa temática devido ao fato dos estudos serem recentes. A maioria das intervenções tem como objetivo modificar a forma de interação entre o cuidador e o paciente, permitindo assim melhores estratégias de gerenciamento de problemas. Consequentemente, há um ajustamento emocional, o que irá impactar na assistência prestada ao paciente ${ }^{33}$.

Ressalta-se que, após terem assumido a responsabilidade de cuidar do familiar idoso, o cuidador necessita ser alvo de orientação dos serviços de saúde, pois é importante ultrapassar as etapas complexas e difíceis muitas vezes referentes aos fatores que incluem aceitação do diagnóstico, manejo com os cuidados diários, lidar com stress diário e administrar o conflito dentro da família e planejar o futuro ${ }^{13}$.

\section{- Espiritualidade}

O estudo que utilizou como método intervenção a teleconferência com a transmissão de mantras para o cuidador, com o objetivo de avaliar a eficácia na aflição psicológica e QV, obteve como resultados uma diminuição na sobrecarga do mesmo ${ }^{25}$. Ressalta-se que nesse estudo houve limitações relacionadas ao tamanho da amostra.

Em outro estudo, dos 53 cuidadores avaliados, $23 \%$ relataram que encontram conforto e amparo na religião ${ }^{22}$.

A literatura tem demostrado através de alguns estudos que há indícios da associação entre QV e espiritualidade/religiosidade. Assim sendo, o campo da QV poderá ser um mediador entre o campo da saúde e assuntos referentes às questões religiosas/espirituais ${ }^{43}$. 


\section{Considerações finais}

O presente estudo permitiu identificar as pesquisas referentes à qualidade de vida dos cuidadores familiares do idoso com demência, bem como de trabalhos abrangentes que contemplem todas as demências e análise das peculiaridades como cada estágio da gravidade pode influenciar na qualidade de vida do cuidador.

As discussões na literatura sobre o ônus que a tarefa de cuidar ocasiona a QV dos cuidadores, assim como o desenvolvimento de estratégias com o intuito de planejar, implementar políticas e programas públicos de suporte social à família, voltados à realidade do cuidador são fundamentais. Atualmente, o idoso e o cuidador convivem com a dependência em uma sociedade que não disponibiliza mecanismos adequados de suporte às famílias.

Nesta perspectiva, a literatura internacional dispõe timidamente de pesquisas que ofereçam estratégias de intervenção, com benefícios cientificamente comprovados. O enfoque na maioria dos estudos foi em cuidadores que prestavam o cuidado a idosos com o diagnóstico de DA, devido a sua prevalência. Porém, torna-se necessário estudos amplos que contemplem as demais demências e as implicações ao cuidador. Detectou-se que a produção científica no Brasil acerca da temática ainda é incipiente, sendo que apenas dois estudos são brasileiros. Entretanto, se faz necessário a rea- lização de estudos de acordo com a realidade brasileira, com a finalidade de propor intervenções que aproximem do contexto de vida do cuidador e do idoso, e colaborem com a definição de políticas públicas na assistência ao idoso com demência.

Os fatores identificados nos estudos que influenciam a qualidade de vida do cuidador familiar do idoso com demência foram: depressão; má qualidade do sono; o tipo de demência do idoso; os sintomas neuropsiquiátricos ocasionados devido a própria doença do idoso; o apoio e suporte social recebido; acesso aos serviços de saúde ; realização de atividades de lazer; problemas de saúde preexistentes; intervenções subsidiadas com treinamento para o cuidador e a espiritualidade relacionada à própria religiosidade.

É importante reconhecer que os estudos aqui descritos enfatizam o suporte educacional, social e psicológico como estratégias de melhoraria da QV destes cuidadores. Portanto, sugere-se que o profissional enfermeiro estabeleça vínculo terapêutico com estas famílias, identifique suas necessidades, para enfim traçar estratégias de cuidado que proporcionem organização e sistematização do cuidado, educação em saúde e suporte social para que, assim, possam superar as dificuldades inerentes a esse processo. Diante do exposto, é primordial o enfoque no cuidador familiar, pois ele é um dos grandes pilares nas intervenções de saúde, sendo inquestionável seu papel no âmbito da família e importância da assistência prestada.

\section{Colaboradores}

LSM Pereira participou da elaboração, levantamento e análise dos dados, resultados e redação do artigo. SM Soares participou de todas as etapas e orientou o trabalho. 


\section{Referências}

1. World Health Organization. Envelhecimento ativo: uma política de saúde. Brasília: Organização Pan-Americana da Saúde; 2005.

2. World Health Organization (WHO). Good health adds life to years: Global brief for World Health Day. Geneva: WHO; 2012.

3. World Health Organization (WHO). Alzheimer's Disease: Some facts and figures. World Health Organization [internet] 2011 [acessado $2014 \mathrm{abr}$ 10]. Disponível em: http://www.searo.who.int/en/Section1174/Section 1199/Section1567/Section1823_806

4. Magaldi RM. Geriatria e Gerontologia Básicas. In: Jacob Filho W, Kikuchi EL, organizadores. Demência. Rio de Janeiro: Elsevier; 2012. p. 221-252.

5. Inouye K, Pedrazzani ES, Pavarini SC, Toyoda CY. Percepção de qualidade de vida do idoso com demência e seu cuidador familiar: avaliação e correlação. Rev. Latino-Am. Enfermagem [periódico na internet]. 2009 [acessado 2014 abr 11]; 17(2) :[cerca de 6 p.]. Disponível em: http://www.revistas.usp.br/rlae/article/viewFile/2559/3139

6. Neto JG,Tamelini MG, Forlenza OV. Diagnóstico diferencial das demências. Rev. Psiq. Clín. [periódico na internet]. 2005; [acessado 2014 jan 28]; 32 (3): [cerca de 11 p.]. Disponível em: http://www.scielo.br/pdf/rpc/ v32n3/a04v32n3.pdf

7. World Health Organization (WHO). The global burden of disease. Geneva: WHO; 2008.

8. Prince M, Acosta D, Chiu H, Scazufca M, Varghese M. Dementia diagnosis in developing countries: a cross-cultural validation study. Lancet 2003; 361(9361):909-917.

9. Burlá C, Camarano AA, Kanso S, Fernandes D, Nunes R. Panorama prospectivo das demências no Brasil: um enfoque demográfico. Cien Saude Colet 2013; 18(10):2949-2956.

10. Giacomin KC, Uchoa E, Lima-Costa MFF. Projeto Bambuí: a experiência do cuidado domiciliário por esposas d idosos dependentes. Cad Saude Publica 2005; 21(5):1509-1518.

11. Grelha PASS. Qualidade Vida dos Cuidadores Informais de Idosos Dependentes em Contexto Domiciliário [dissertação]. Lisboa: Universidade de Lisboa Faculdade de Medicina de Lisboa; 2009.

12. Anjos KF, Boery RNSO, Pereira R. Qualidade de vida de cuidadores familiares de idosos dependentes no domicílio. Texto Contexto Enferm [periódico na internet]. 2014, [acessado $2014 \mathrm{dez}$ 20]; 23(3): [cerca de 8 p.]. Disponível em: http://www.scielo.br/pdf/tce/v23n3/ pt_0104-0707-tce-23-03-00600.pdf

13. Caldas CP. Envelhecimento com dependência: responsabilidades e demandas da família. Cad Saude Publica 2003; 19(3):773-781.

14. FernandesMGM, Garcia TR. Determinantes da tensão do cuidador familiar de idosos dependentes. Rev. bras. enferm. [periódico na internet]. 2009, [acessado $2014 \mathrm{dez}$ 20]; 63(1): [cerca de 6 p.]. Disponível em: http://www.scielo.br/scielo.php?script=sci_arttext\&pi$\mathrm{d}=$ S0034-71672009000100009
15. Mendes KDS, Silveira RCCP, Galvão CMR. Revisão Integrativa: Método de pesquisa para a incorporação de evidências na saúde e na enfermagem. Texto Contexto Enferm [periódico na internet]. 2008; [acessado 2014 fev 10]; 17(4): [cerca de 6 p.]. Disponível em: www. scielo.br/pdf/tce/v17n4/18.pdf

16. Ursi ES. Prevenção de lesão de pele no perioperatório: uma revisão integrativa [dissertação]. Ribeirão Preto: Escola de Enfermagem de Ribeirão Preto; 2005.

17. Stetler CB, Norsi D, Ruscki S, Broughton S, Corrigan B, Fitzgeralg Z, Giuliano K, Havener P, Sheridan EA. Utilization-Focused Integrative Reviews in a Nursing service. Appl Nurs Res 1998; 11(4):195-206.

18. Karsch U M. Idosos dependentes: famílias e cuidadores. Caderno Saúde Pública. [periódico na internet]. 2003; [acessado 2014 jan 20]; 19(3): [cerca de 5 p.]. Disponível em: http://www.scielosp.org/pdf/csp/v19n3/15890. pdf.

19. Mazza MMPR, Lefèvre F. Cuidar em família: análise da representação social da relação do cuidador familiar com o idoso. Rev Bras Cresc Desenv Hum [periódico na internet]. 2005; [acessado 2014 jan 25];15(1): [cerca de 9 p.]. Disponível em: http://pepsic.bvsalud.org/pdf/ $\mathrm{rbcdh} / \mathrm{v} 15 \mathrm{n} 1 / 02 . \mathrm{pdf}$

20. Moraes EN. Atenção á saúde do idoso: aspectos conceituais. Brasília: Organização Pan-Americana da Saúde; 2012.

21. Kuo LM, Huang HL, Huang HL, Liang J, Chiu YC, Chen ST, Kwok YT, Hsu WC, Shyu YI. A home-based training program improves Taiwanese Family caregivers' quality of life and decreases their risk for depression: a randomized controlled trial. Int J Geriatr Psychiatry 2013; 28(5):504-513.

22. Keika I, Pedrazzani ES, Pavarini SCI. Implicações da doença de Alzheimer na qualidade de vida do cuidador: um estudo comparativo. Cad Saude Publica 2010; 26(5):891-899.

23. Chien WT, Lee YM. A disease management program for families of persons in hong kong with dementia. Psychiatr Serv 2008; 59(4):433-436.

24. Tomomitsu MRS, Perracini MR, Neri AL. Fatores associados à satisfação com a vida em idosos cuidadores e não cuidadores. Cien Saude Colet 2014; 19(8):34293440.

25. Bormann J, Kathleen A, Warren MS, Regalbuto L, Glaser D, Kelly A, Schnack J, Hinton L. A spiritually based caregiver intervention with telephone delivery for family caregivers of veterans with dementia. Fam Community Health [serial on the Internet] 2009; [cited 2014 mar 16]; 32(4): [about 8 p]. Available from: http:// ot.creighton.edu/community/EBLP/Question5/2010_ Update/Bormann\%202009.pdf

26. Riedijk SR, Vugt ME, Duivenvoorden HJ, Niermeijer MF, Swieten VJC, Verhey FRJ, Tibben A. Caregiver burden, health-related quality of life and coping in dementia caregivers: a comparison of Fronto Temporal Dementia and Alzheimer's disease. Dement Geriatr Cogn Disord. [serial on the Internet]. 2006; [cited 2014 abr 01]; 22: [about 7 p.]. Available from: http://www. karger.com/Article/FullText/95750 
27. Takai M, Takahashi M, Iwamitsu Y, Oishi S, Miyaoka $\mathrm{H}$. Subjective experiences of family caregivers of patients with dementia as predictive factors of quality of life. Psychogeriatrics [serial on the Internet]. 2011; [cited 2014 mar 15]; 11: [about 6 p.]. Available from: http://onlinelibrary.wiley.com/doi/10.1111/j.14798301.2011.00354.x/pdf

28. Cupidi C, Realmuto S, Lo Coco G, Cinturino A, Talamanca S, Arnao V, Perini V, Piccoli T, D'Amelio M, Savettieri G, Lo Coco D. Sleep quality in caregivers of patients with Alzheimer's disease and Parkinson's disease and its relationship to quality of life. Int Psychogeriatr 2012; 24(11):1827-1835.

29. Bartfay E, Bartfay W. Quality-of-Life outcomes among Alzheimer's disease family caregivers following community-based intervention. West J Nurs Res 2011; 35(1):98-116.

30. Borghi AC, Sassá AH, Matos PCB, Decesaro MN, Marcon SS. Qualidade de vida de idosos com doença de Alzheimer e de seus cuidadores. Rev Gaúcha Enferm. [periódico na internet].2011; [acessado 2014 mar 05]; 32(4): [cerca de 7p.]. Disponível em: http://seer.ufrgs. br/RevistaGauchadeEnfermagem/article/view/21593

31. Kuzu N, Beser N, Zencir M, Sahiner T, Ahiner, MD, Nesrin E, Ahmet E, Binali C, Cagdaş E. Effects of a comprehensive educational program on quality of life and emotional issues of dementia patient caregivers. Geriatr Nurs 2005; 26(6):378-386.

32. Llacha BN, Suriñachb L, Gamisansb MR. Calidad de vida, tiempo de dedicación y carga percibida por el cuidador principal informal del enfermo de Alzheimer. Aten Primaria [periódico na Internet]. 2004; [acessado 2014 mar 23]; 34(4): [cerca de 7 p.]. Disponível em: http://www.sciencedirect.com/science/article/pii/S0 212656704789040

33. Cruz MN, Lecheta DR, Wachholz PA. Fatores associados à sobrecarga e à depressão em cuidadores de idosos com doença de Alzheimer. Geriatria \& Gerontologia [periódico na internet].2009; [acessado 2014 maio 07]; 3(1): [cerca de 8 p.]. Disponível em: http://www.sbgg. org.br/profissionais/arquivo/revista/volume3-numerol/artigo04.pdf

34. Alzheimer Disease International (ADI). Dementia and risk reduction. London: ADI; 2014.

35. Luzardo AR, Gorini MIPC, Silva APSS. Características de idosos com doença de Alzheimer e seus cuidadores: uma série de casos em um serviço de neurogeriatria. Texto Contexto Enferm [periódico na internet]. 2006; [acessado 2014 maio 22]; 15(4): [cerca de 7 p.]. Disponível em: http://www.scielo.br/pdf/tce/v15n4/v15n4 a06.pdf

36. Gutierrez BAO, Silva HS, Guimarães C, Campino AC Impacto econômico da doença de Alzheimer no Brasil: é possível melhorar a assistência e reduzir custos? Cien Saude Colet 2014; 19(11):4479-4486.
37. Fernandes MTO, Soares SM. O desenvolvimento de políticas públicas de atenção ao idoso no Brasil. Rev. esc. enferm. USP [periódico na internet]. 2012; [acessado 2014 maio 20];46(6): [cerca de 8 p.]. Disponível em: http://www.scielo.br/scielo.php?script=sci_pdf\&pid $=$ S008062342012000600029\&lng $=$ en \&nrm $=$ iso\&tln$\mathrm{g}=\mathrm{pt}$

38. Cattani RB, Girardon-Perlini NMO. Cuidar do idoso doente no domicílio na voz de cuidadores familiares. Rev Eletrônica de Enfermagem [periódico na internet]. 2004; [acessado 2014 maio 1];6(2): [cerca de 7 p.].Disponível em: http://www.revistas.ufg.br/index.php/fen/ article/view/812/929

39. Santos AA, Pavarini SCI. Perfil dos cuidadores de idosos com alterações cognitivas em diferentes contextos de vulnerabilidade social. Rev Gaúcha Enferm [periódico na internet]. 2010; [acessado 2014 maio 1]; 31(1): [cerca de 7 p.].Disponível em: http://seer.ufrgs.br/RevistaGauchadeEnfermagem/article/view/9430

40. Vitaliano PP, Young HM, Zhang J. Is caregiving a risk factor for illness? Current Directions in Psychological Science 2004; 13(1):13-16.

41. Grunfeld E, Coyle D, Whelan T, Clinch J, Reyno L, Earle CC, Willan A, Viola R, Coristine M, Janz T, Glossop R. Family caregiver burden: results of a longitudinal study of breast cancer patients and their principal caregivers. CMAJ [periódico na internet] 2004; [acessado 2014 maio 1]; 170(12): [cerca de 6 p.].Disponível em: http://www.cmaj.ca/content/170/12/1795.full.pdf

42. Martins JJ, Albuquerque GL, Nascimento ERP, Barra DCC, Souza WGA, Pacheco WNS. Necessidades de educação em saúde dos cuidadores de pessoas idosas no domicílio. Texto Contexto Enferm. [periódico na internet]. 2007; [acessado 2014 maio 15] ;16(2): [cerca de 8 p.].Disponível em: http://www.scielo.br/pdf/tce/ v16n2/a07v16n2.pdf

43. Panzini RG. Rocha NS,Bandeira DR, Fleck MPA. Qualidade de vida e espiritualidade Rev. Psiq. Clín. [periódico na internet]. 2007; [acessado 2014 maio 16]; 34: [cerca de 10 p.]. Disponível em: http://www.scielo.br/ pdf/rpc/v34s1/a14v34s1.pdf.

Artigo apresentado em 30/06/2014

Aprovado em 26/01/2015

Versão final apresentada em 28/01/2015 
